\title{
Estudo da reabsorção radicular apical após o uso de aparelho extrabucal no tratamento da má oclusão do tipo Classe II, $1^{a}$ divisão dentária
}

Vânia Célia Vieira de Siqueira*, Gustavo Hauber Gameiro**, Maria Beatriz Borges Araújo Magnani***,

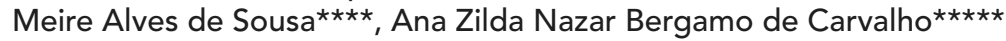

\section{Resumo}

Objetivo: o presente estudo verificou o efeito do uso do aparelho extrabucal (AEB) sobre a intensidade da reabsorção radicular apical nos primeiros molares permanentes superiores submetidos à ação do aparelho. Métodos: foram avaliadas as radiografias periapicais da região dos primeiros molares permanentes superiores, bilateralmente, de 19 jovens leucodermas, do gênero feminino, com idades entre 8 e 10 anos, com má oclusão do tipo Classe II, $1^{\text {a }}$ divisão dentária, pré e pós-tratamento com AEB de tração alta. As 76 radiografias foram divididas em dois grupos, de acordo com o grau de formação radicular. O grupo A consistiu de 18 radiografias com formação radicular incompleta, com exceção da raiz palatina, ao início do tratamento e 18 ao final. O grupo B consistiu de 20 radiografias com formação radicular completa ao início do tratamento e 20 ao final. Mensurou-se os comprimentos radiculares utilizando um paquímetro digital e submeteu-se as medidas obtidas ao teste de erro do método e à análise estatística (teste $t$ de Student) para verificar as diferenças no comprimento radicular antes e após o tratamento com o AEB. Resultados: no grupo A ocorreu um aumento significativo dos comprimentos radiculares, enquanto no grupo B as diferenças dos comprimentos radiculares não foram significativas. As medidas pós-tratamento no grupo A não diferiram das medidas pré-tratamento no grupo B, ou seja, os dentes com formação radicular incompleta ao início do tratamento apresentaram crescimento radicular normal durante o tratamento ativo. Conclusão: concluiu-se que o uso do AEB não influenciou negativamente na formação radicular e não provocou reabsorção apical nos molares submetidos à ação do aparelho, sugerindo que o AEB não apresentou riscos à estrutura e formação radicular quando corretamente indicado e aplicado.

Palavras-chave: Reabsorção radicular apical. Aparelho extrabucal. Classe II, $1^{\text {a }}$ divisão dentária.

\footnotetext{
* Professora doutora da disciplina de Ortodontia da Faculdade de Odontologia de Piracicaba/UNICAMP. Professora adjunta III da Disciplina de Ortodontia da Pontifícia Universidade Católica de Minas Gerais - PUC/Minas.

** Doutor em Ortodontia pela Faculdade da Odontologia de Piracicaba/UNICAMP.

*** Professora doutora da disciplina de Ortodontia da Faculdade de Odontologia de Piracicaba/UNICAMP.

**** Aluna de doutorado em Ortodontia da Faculdade de Odontologia de Piracicaba/UNICAMP.

$\star \star \star \star \star$ Mestre em Ortodontia pela Faculdade de Odontologia de Piracicaba/UNICAMP.
} 


\section{INTRODUÇÃO}

A reabsorção radicular apical situa-se entre os mais comuns e indesejáveis efeitos colaterais do tratamento ortodôntico ${ }^{2,3,4,8,31}$. O encurtamento radicular resultante da reabsorção radicular apical externa é irreversível e difícil de prever ${ }^{23}$. Como características, essa reabsorção, geralmente suave, mostra-se assintomática e preserva a vitalidade pulpar ${ }^{7}$, sem alteração na coloração dos dentes. Do ponto de vista histológico, a atividade osteoblástica compensa a redução tecidual, à medida que se processa a perda radicular, conferindo à imagem radiográfica a integridade do espaço periodontal. Em consequência, os dentes mantêm-se estáveis ${ }^{25}$ e sem mobilida$\mathrm{de}^{33}$, não retratam importância clínica maior ${ }^{14}$ e o prognóstico mostra-se geralmente favorável. Uma vez removida a força ortodôntica, cessa o processo de reabsorção ${ }^{13}$ e ocorre deposição de cemento secundário, contornando e reparando a morfologia radicular ${ }^{15,23}$.

No entanto, alguns pacientes mostram-se mais severamente afetados pelas reabsorções radiculares ${ }^{6}$. Quando mais da metade do comprimento radicular encontra-se perdido por reabsorção, a função e a estabilidade dos dentes afetados apresentam-se seriamente comprometidas ${ }^{9}$.

Os fatores de risco capazes de contribuir com a reabsorção radicular externa incluem a predisposição genética ${ }^{1,19}$, o gênero do paciente $^{13}$, os desvios na forma radicular ${ }^{12}$, os dentes traumatizados previamente ao tratamento ortodôntico ${ }^{14}$, os dentes tratados endodonticamen$\mathrm{te}^{32}$, os hábitos nocivos (como a onicofagia) ${ }^{21}$, a idade do paciente, o estágio de formação radicular ao início do tratamento ortodôntico ${ }^{13}$, o tipo de aparelho ortodôntico utilizado ${ }^{5,17,26,27}$, as forças aplicadas ${ }^{10}$ e a duração do tratamento ${ }^{12}$. Além disso, considera-se a capacidade individual do profissional na aplicação de qualquer técnica ortodôntica, bem como a utilização de elásticos intermaxilares, molas de forças variadas, uso de fios redondos ou retangulares, velocidade da retração anterior e o uso ou não de ancoragem extrabucal ${ }^{17,18,24}$.

O interesse crescente sobre informações mais esclarecedoras em relação à reabsorção radicular apical ocorreu, principalmente, por duas razões: o mapeamento do genoma humano, transmitindo a ideia de que poderia auxiliar em um melhor entendimento do processo patológico ${ }^{1}$; e o número crescente de processos legais contra os ortodontistas $^{28}$. Do ponto de vista legal, a situação torna-se embaraçosa para o profissional e, se nenhum tipo de conduta preventiva foi adotada durante o tratamento - com a finalidade de controlar esse risco -, sua conduta poderia ser caracterizada como negligência. O que se observa dos inúmeros estudos na literatura é a advertência de um exame radiográfico periapical prévio, como rotina de diagnóstico e ao longo do tratamento, associado a uma anamnese acurada e um adequado controle da mecanoterapia ${ }^{17,24,30}$.

Atualmente, os profissionais preocupam-se pouco com as possíveis reabsorções radiculares nos dentes posteriores, provavelmente pela reabsorção na região anterior mostrar-se mais acentuada $^{29}$ e pela maior dificuldade técnica em constatar a reabsorção nos molares. No entanto, esses dentes também mostram-se vulneráveis à mecanoterapia e vários estudos demonstram que a intensidade da reabsorção radicular nesses dentes mostra-se similar à dos incisivos ${ }^{16,22,30}$. Além disso, pouco se sabe sobre a quantidade de reabsorção radicular nos molares decorrente do uso de aparelhos extrabucais, comumente empregados durante a mecanoterapia de Classe II.

\section{PROPOSIÇÃO}

Apoiados nas premissas acima, os autores deste trabalho propuseram-se a avaliar a ocorrência ou não da reabsorção radicular apical externa nos primeiros molares superiores, em pacientes com má oclusão do tipo Classe II, $1^{\text {a }}$ divisão dentária, após 12 meses de tratamento com aparelho extrabucal de tração alta. 


\section{MATERIAL E MÉTODOS Composição e critérios de seleção da amostra}

O presente trabalho iniciou-se somente após a obtenção de aprovação junto ao Comitê de Ética em Pesquisa da Faculdade de Odontologia de Piracicaba (UNICAMP).

Para a seleção da amostra, examinou-se aproximadamente 700 jovens da rede pública de ensino - nível fundamental, da cidade de Piracicaba/ $\mathrm{SP}$ - do gênero feminino, leucodermas, com idades variando entre 8 e 10 anos, que nunca tivessem sido submetidas a tratamento ortodôntico prévio. Com a devida autorização do responsável legal, mediante Termo de Consentimento Livre e Esclarecido, selecionou-se 19 jovens, com má oclusão do tipo Classe II, $1^{\text {a }}$ divisão dentária.

Em ficha especialmente elaborada para a presente pesquisa, anotou-se os dados pessoais, bem como as características oclusais relativas a cada jovem selecionada. Durante o exame clínico, observou-se:

1) Primeiros molares permanentes e caninos decíduos superiores posicionados mesialmente em relação aos primeiros molares permanentes e caninos decíduos inferiores, respectivamente.

2) Trespasse horizontal entre os incisivos superiores e inferiores maior que $3 \mathrm{~mm}$.

Para diagnosticar adequadamente os grupos, solicitou-se, ainda, de cada jovem selecionada, exames complementares, que consistiram de:

- Telerradiografias, obtidas em norma lateral, radiografias panorâmicas e radiografias periapicais da região dos primeiros molares permanentes superiores, bilateralmente.

- Modelos de estudo em gesso.

- Fotografias intra e extrabucais.

Cefalometricamente, as jovens apresentavam padrão de crescimento vertical com valores médios de SN.GoGn em $35^{\circ}$ e de FMA em $27^{\circ}$, dimensão maxilar média em $50 \mathrm{~mm}$, dimensão mandibular média em $95 \mathrm{~mm}$, SNA médio em $80^{\circ}$, SNB médio em $75^{\circ}$, ANB médio em $5^{\circ}$.

As jovens receberam tratamento ortodôntico empregando aparelho extrabucal, com tração alta, com força de $250 \mathrm{~g}$ no primeiro mês de tratamento. Ao final desse período, a força passou para $350 \mathrm{~g}$ e uso diário de 14-16 horas. Após a obtenção da relação oclusal dos primeiros molares em normalidade, reduziu-se a força para $250 \mathrm{~g}$ por três meses e, ao final desse período, encerrou-se o tratamento ortodôntico com o AEB. O tempo médio de duração do tratamento ortodôntico foi de 12 meses.

As radiografias periapicais foram divididas em dois grupos, de acordo com o grau de formação radicular. $\mathrm{O}$ grupo $\mathrm{A}$ consistiu de 18 radiografias com formação radicular incompleta, estágio 8 de Nolla ${ }^{20}$ - com exceção da raiz palatina, que encontrava-se formada -, ao início do tratamento ortodôntico e 18 radiografias periapicais ao final do tratamento. $\mathrm{O}$ grupo B consistiu de 20 radiografias com formação radicular completa ao início do tratamento ortodôntico e 20 radiografias periapicais ao final do tratamento.

\section{Radiografias}

As radiografias periapicais pré e pós-tratamento foram obtidas por um único operador, com um aparelho de raios X DABI 70 Spectro 1070 X (Dabi Atlante, Ribeirão Preto/SP), regulado para $70 \mathrm{KV}$ e $10 \mathrm{~mA}$, com a técnica do paralelismo, cone longo. Os filmes utilizados foram EktaSpeed EP 21 (Kodak, EUA), expostos por 0,9 segundos. Todas as radiografias foram processadas automaticamente.

\section{Quantificação da reabsorção radicular apical}

Sobre cada radiografia periapicial, adaptou-se uma folha de papel de acetato tipo Ultraphan, com espessura de $63 \mathrm{~g} / \mathrm{m}^{3}$. Utilizou-se um negatoscópio em sala obscurecida para facilitar a visualização das estruturas anatômicas de interesse ao estudo. Identificou-se manualmente o primeiro molar permanente, a coroa e suas raízes mesiovestibular (MV), distovestibular (DV) e palatina (P) com o auxílio de uma lapiseira do tipo Pentel com grafite de $0,3 \mathrm{~mm}$ de espessura. 
Mensurou-se os comprimentos: dos ápices das raízes mesiovestibulares até as pontas das cúspides mesiovestibulares, dos ápices das raízes distovestibulares até as pontas das cúspides distovestibulares e dos ápices das raízes palatinas até as pontas das cúspides distopalatinas dos primeiros molares superiores, utilizando um paquímetro digital (Lee Tools ${ }^{\circledR}$,USA) nos traçados realizados nas radiografias periapicais (Fig. 1, 2).

O mesmo investigador realizou os traçados duas vezes, com intervalo de 30 dias, obtendo os valores que foram submetidos ao teste do erro do método, utilizando a fórmula de Dalbergh ${ }^{11}$. Após essa fase, os valores médios iniciais e finais foram submetidos à avaliação estatística empregando-se o teste $t$ de Student para verificar possíveis diferenças com relação ao comprimento radicular antes e após o tratamento com AEB.

\section{Análise estatística}

Com a intenção de avaliar o grau de concordância intraexaminador, instituiu-se a medição do grau da reprodutibilidade dos traçados e, consequentemente, das mensurações, utilizando a fórmula de Dahlberg, preconizada por Houston ${ }^{11}$, em 1983. Foram escolhidas, aleatoriamente, as mensurações de 10 radiografias para a aquisição de valores para o cálculo do erro do método, obtendo-se o valor de $0,29 \mathrm{~mm}$, indicando ausência de erro na metodologia aplicada.

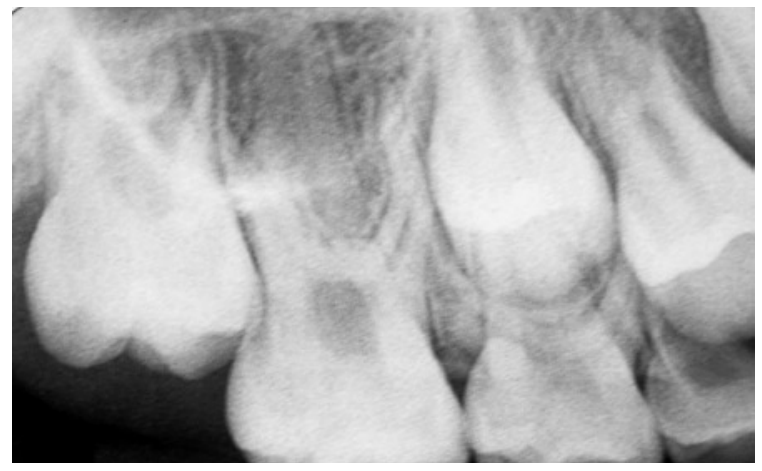

FIGURA 1 - Radiografia periapical da região do primeiro molar permanente ao início do tratamento com o AEB.
Para verificar se existiam diferenças com relação ao comprimento radicular antes e após o tratamento e entre os grupos avaliados, tratado e controle, aplicou-se o teste $t$ de Student, com nível de significância em $\mathrm{p}<0,05$.

\section{RESULTADOS}

Mensurações dos comprimentos radiculares nas radiografias periapicais pré e pós-tratamento com AEB no grupo A (formação radicular incompleta)

No grupo A, com formação radicular incompleta, os valores médios dos comprimentos radiculares dos molares superiores, direito e esquerdo, obtidos nas radiografias periapicais pré e pós-tratamento, apresentaram um aumento significativo (alongamento radicular) após 12 meses de uso do $\mathrm{AEB}$, com exceção da raiz palatina, que não sofreu alteração (Gráf. 1).

\section{Mensurações dos comprimentos radiculares nas radiografias periapicais pré e pós-trata- mento com AEB no grupo B (formação radi- cular completa)}

No grupo B, com formação radicular completa, os valores médios dos comprimentos radiculares dos molares superiores, direito e esquerdo, obtidos nas radiografias periapicais pré e pós-tratamento não diferiram estatisticamente após 12 meses de uso do AEB (Gráf. 2).

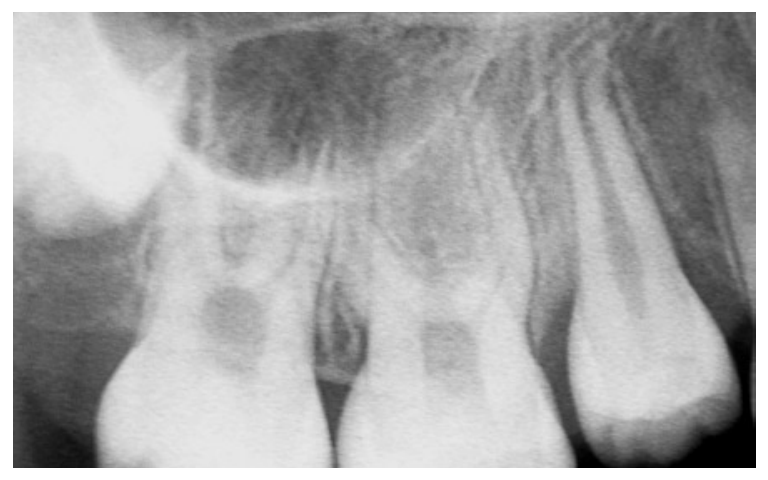

FIGURA 2 - Radiografia periapical da região do primeiro molar permanente, do mesmo caso, ao final do tratamento com o AEB. 


\section{Comparações entre os comprimentos radicu- lares pós-tratamento no grupo A e os com- primentos radiculares pré-tratamento no grupo B}

Os valores médios dos comprimentos radiculares pós-tratamento no grupo A não diferiram estatisticamente dos comprimentos radiculares pré-tratamento no grupo $\mathrm{B}$, com exceção da raiz $M V$, que apresentou um aumento estatisticamente significativo em relação ao comprimento prétratamento da raiz MV no grupo B (Gráf. 3).

\section{Comprimentos radiculares no grupo A (forma- ção radicular incompleta) e grupo B (formação}

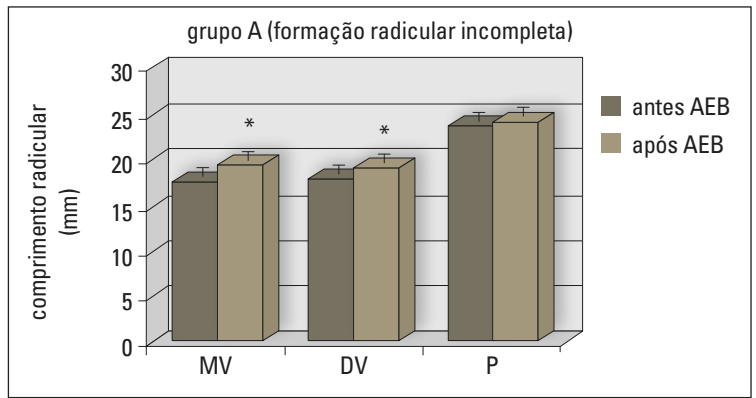

GRÁFICO 1 - Média dos comprimentos radiculares das raízes mesiovestibular (MV), distovestibular (DV) e palatina (P) dos primeiros molares superiores antes e após o uso do AEB (* indica diferença estatística entre as médias pré e pós-tratamento) ( $\mathrm{p}<0,05$, teste t pareado).

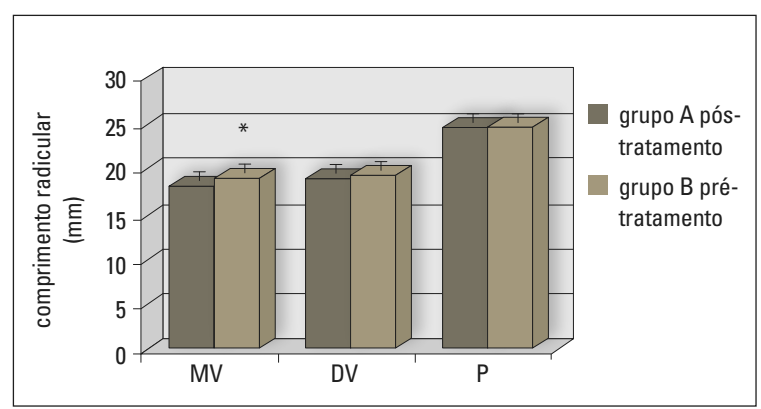

GRÁFICO 3 - Média dos comprimentos radiculares das raízes mesiovestibular (MV), distovestibular (DV) e palatina (P) dos primeiros molares superiores no grupo A pós-tratamento e grupo B pré-tratamento ( $^{*}$ indica diferença estatística entre os grupos $A$ e B) $(p<0,05$, teste $t)$. radicular completa) obtidos nas radiografias periapicais pré e pós-tratamento com AEB

A tabela 1 apresenta os valores médios dos comprimentos das raízes mesiovestibular (MV), distovestibular (DV) e palatina (P) no grupo A formação radicular incompleta; e grupo B - formação radicular completa (amostra total).

\section{DISCUSSÃO}

Verifica-se, na última década, várias revisões de literatura publicadas sobre o tema reabsorção radicular ${ }^{2,3,8}$. Durante aproximadamente 30 anos, o norueguês Kaare Reitan foi considerado um dos maiores pesquisadores da microscopia

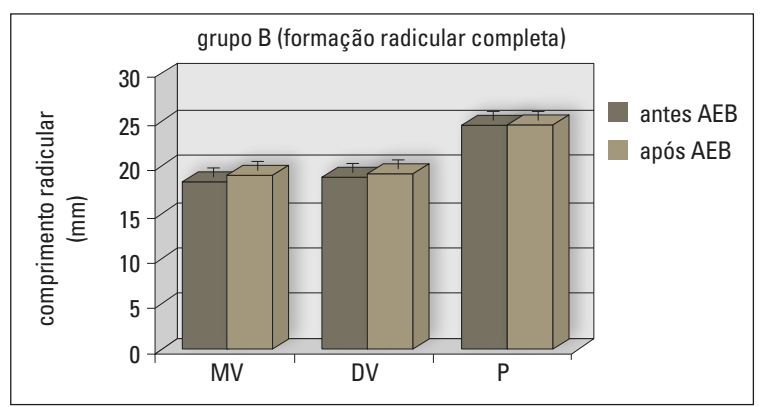

GRÁFICO 2 - Média dos comprimentos radiculares das raízes mesiovestibular (MV), distovestibular (DV) e palatina (P) dos primeiros molares superiores antes e após o uso do AEB. Não ocorreu diferença estatística entre as médias pré e pós-tratamento ( $p<0,05$, teste t pareado).

TABELA 1 - Médias dos comprimentos radiculares com os desvios-padrão $(\mathrm{mm})$ no grupo $A$ (formação radicular incompleta) e no grupo B (formação radicular completa).

\begin{tabular}{|c|c|c|c|c|}
\hline & \multicolumn{2}{|c|}{ grupo A } & \multicolumn{2}{|c|}{ grupo B } \\
\hline & $\begin{array}{l}\text { antes } \\
\text { AEB }\end{array}$ & $\begin{array}{l}\text { após } \\
\text { AEB }\end{array}$ & $\begin{array}{l}\text { antes } \\
\text { AEB }\end{array}$ & $\begin{array}{l}\text { após } \\
\text { AEB }\end{array}$ \\
\hline $\begin{array}{l}\text { raiz mesioves- } \\
\text { tibular (MV) }\end{array}$ & $17,4 \pm 1,8$ & $19,4 \pm 1,6^{*} \#$ & $18,3 \pm 1,2$ & $18,7 \pm 1,5$ \\
\hline $\begin{array}{l}\text { raiz distovesti- } \\
\text { bular (DV) }\end{array}$ & $17,8 \pm 1,5$ & $18,9 \pm 1,7^{*}$ & $18,7 \pm 1,2$ & $19,2 \pm 1,3$ \\
\hline $\begin{array}{c}\text { raiz } \\
\text { palatina }(P)\end{array}$ & $23,5 \pm 1,1$ & $23,9 \pm 1,0$ & $24,4 \pm 1,0$ & $24,4 \pm 1,0$ \\
\hline
\end{tabular}

* indica diferença estatística dentro de cada grupo, entre os valores pré e pós-tratamento ( $p<0,05$, teste t pareado). \# indica diferença estatística entre os grupos $A$ pós-tratamento e $B$ pré-tratamento ( $p<0,05$, teste $t)$. 
da movimentação dentária induzida. No ano de 1957, o referido autor analisou a variação individual dos tecidos, o tipo de força aplicada e os princípios mecânicos da movimentação ortodôntica ${ }^{24}$. Os resultados permitiram elucidar a existência de um período inicial necessário, após a aplicação da força, para atingir um estado de proliferação celular e promover a alteração tecidual. Este estágio depende de uma série de fatores, incluindo a idade do paciente e as características anatômicas da raiz. Com relação ao tipo de força aplicada, o autor distinguiu as forças do tipo intermitente e contínua. Desde então, realizam-se diversos estudos clínicos e radiográficos, com diferentes metodologias, relacionando a reabsorção radicular apical às diversas técnicas de tratamento ortodôntico.

A literatura pertinente oferece dados e evidências de alguns aspectos importantes relacionados ao maior ou menor grau de reabsorção radicular. Os movimentos dentários de inclinação, mecânica intrusiva, tratamento com extrações dentárias e técnicas ortodônticas que aplicam forças intensas encontram-se, frequentemente, associados à reabsorção dentária ${ }^{3}$.

Apesar dos registros científicos revelarem uma busca pelas causas que poderiam instigar o processo de reabsorção radicular durante o tratamento ortodôntico, hoje sabe-se que a reabsorção radicular possui uma etiologia multifatorial resultante de uma interação complexa entre a biologia individual e o efeito das forças mecânicas ${ }^{3}$. A procura constante dos ortodontistas por uma técnica que minimize esse efeito deletério induziu a busca pelo esclarecimento das consequências reais e o estabelecimento de um maior domínio na utilização de cada aparelho, aumentando a previsibilidade de seus resultados ${ }^{17}$.

Em relação ao aparelho extrabucal (AEB), utilizado geralmente na fase de dentadura mista e início da permanente, o presente estudo questionou a interferência que esse aparelho ocasionaria sobre as estruturas dentárias radiculares. Portanto, tornou-se relevante a avaliação dos efeitos do AEB sobre a estrutura radicular dos primeiros molares superiores que receberam as forças aplicadas pelo referido aparelho.

Os resultados da presente pesquisa demonstraram que o AEB não influenciou negativamente na formação radicular e não provocou reabsorção apical externa dos molares submetidos à sua ação. Inicialmente, dividiu-se as radiografias periapicais das jovens com má oclusão do tipo Classe II, $1^{\text {a }}$ divisão dentária, em dois grupos: o grupo A consistiu de 36 radiografias com formação radicular incompleta, com exceção da raiz palatina, e o grupo B consistiu de 40 radiografias com formação radicular completa. Essa estratégia possibilitou a avaliação tanto da reabsorção radicular propriamente dita (diferenças entre os valores pré e pós-tratamento no grupo B) como do crescimento radicular (diferenças entre os valores pré e pós-tratamento no grupo A) nas jovens que receberam tratamento ortodôntico utilizando o AEB de tração alta por um período médio de 12 meses.

No grupo A, com formação radicular incompleta, os aumentos dos comprimentos radiculares foram estatisticamente significativos, quando comparadas as médias dos valores pré e pós-tratamento com AEB. Neste grupo, apenas o comprimento da raiz palatina após o uso do AEB não diferiu estatisticamente do seu comprimento antes do tratamento, visto que essa raiz encontrava-se, ao início do tratamento, em fase de fechamento do ápice radicular. Por outro lado, no grupo B, com formação radicular completa, os valores médios dos comprimentos radiculares dos molares superiores não diferiram estatisticamente após o uso do AEB. Esse resultado indicou que o AEB não provocou qualquer grau significativo de reabsorção apical nas raízes dos molares superiores submetidos à sua ação.

A comparação entre os comprimentos radiculares pós-tratamento no grupo A e os comprimentos radiculares pré-tratamento no grupo B demonstrou que os dentes com formação radicular incompleta ao início do tratamento alcançaram a 
média normal do comprimento radicular. Os valores médios dos comprimentos radiculares pós-tratamento no grupo A não diferiram estatisticamente dos comprimentos radiculares pré-tratamento no grupo B, com exceção da raiz MV, que apresentou um aumento estatisticamente significativo em relação ao comprimento pré-tratamento da raiz MV no grupo B. Esse resultado indicou que o AEB não influenciou negativamente no crescimento radicular. O maior comprimento da raiz MV no grupo A pós-tratamento em relação ao grupo B prétratamento pode ser explicado pelo fato de que os dentes com formação radicular incompleta possuem uma maior proteção ou resistência contra a reabsorção radicular, quando comparados com os dentes que já possuem suas raízes formadas ${ }^{9}$.

O estudo de Hendrix et al. ${ }^{9}$, em 1994, avaliou o grau de reabsorção radicular em dentes posteriores de pacientes ortodônticos, os quais foram divididos em grupos com e sem formação radicular completa. Os dentes posteriores apresentaram uma redução significativa nos comprimentos radiculares durante o tratamento ativo, independentemente do gênero, idade, terapia (com ou sem extração) e duração do tratamento ativo. Além disso, os dentes com formação radicular incompleta apresentaram um aumento significativo nos comprimentos radiculares após o tratamento, porém esse aumento não alcançou a média normal dos comprimentos radiculares observados no grupo com raízes formadas ao início do tratamento 9 . Os resultados do presente estudo não demonstraram grau significativo de reabsorção radicular em nenhum dos grupos estudados, e até mesmo um aumento radicular foi observado no comprimento pós-tratamento da raiz MV que encontrava-se em formação, em relação ao comprimento pré-tratamento da raiz MV que encontrava-se formada. É importante enfatizar que o presente estudo avaliou o grau de reabsorção radicular após 12 meses de tratamento com AEB, enquanto o trabalho de Hendrix et al. ${ }^{9}$ avaliou a reabsorção radicular após o tratamento ortodôntico com a técnica de Edgewise padrão, usando fios retangulares, com duração do tratamento de aproximadamente 2 anos.

Frequentemente, indica-se o uso do AEB durante a fase de dentadura mista e início da permanente, época em que as raízes dos molares superiores podem não se encontrar completamente formadas. Nossos resultados não apontam riscos à formação radicular durante o uso do AEB, o que favorece a indicação de iniciar o tratamento ortodôntico em pacientes com idade precoce, a qual representa o estágio mais ativo de desenvolvimento dentário e crescimento esquelético craniofacial $^{18}$. Os dados deste estudo concordam com os estudos radiográficos de Rudolph ${ }^{27}$, Rosenberg ${ }^{26}$, Linge e Linge ${ }^{13}$, bem como com os estudos histológicos de Reitan ${ }^{24}$, todos favorecendo o tratamento precoce.

O uso do AEB pode até mesmo reduzir os riscos de reabsorção radicular apical, conforme demonstrado por Brin et $\mathrm{al}^{4}{ }^{4}$, em 2003, que demonstraram que pacientes tratados em duas fases com o AEB ou bionator, seguido por aparelhos fixos, apresentaram menor grau de reabsorção radicular, enquanto os pacientes tratados em uma fase, com os aparelhos fixos, demonstraram a maior quantidade de reabsorção. Segundo os autores, os pacientes que se submeteram a tratamento com aparelhos ortopédicos necessitaram de menor correção da sobressaliência, durante a fase de aparelhos fixos ${ }^{4}$. Os resultados obtidos corroboram a segurança e eficiência do uso do AEB em pacientes com má oclusão do tipo Classe II, $1^{\text {a }}$ divisão dentária, na fase da dentadura mista.

\section{CONCLUSÕES}

Baseado na amostra estudada, na metodologia empregada durante o desenvolvimento do presente estudo e de acordo com os resultados apresentados, parece-nos lícito concluir que:

1) No grupo que apresentou os molares superiores com formação radicular completa, não ocorreu reabsorção radicular apical após 12 meses de uso do AEB. 
2) No grupo que apresentou os molares superiores com formação radicular incompleta, o crescimento radicular ocorreu normalmente durante os 12 meses de uso do AEB.

3) O AEB não apresentou riscos à estrutura e formação radicular quando corretamente indicado e aplicado.

\title{
Study of apical root resorption after occipital headgear wear on the treatment of dental Class II, division 1 malocclusion
}

\begin{abstract}
Aim: The aim of this study was to verify the effect of occipital headgear wear on the intensity of apical root resorption of the upper first permanent molars submitted to the action of the appliance. Methods: Were evaluated periapical X-rays of 19 leucoderms young female - with ages between 8 and 10 years, with dental Class II, division 1 malocclusion, before and after orthodontic treatment, using high-pull headgear. The $76 \mathrm{X}$-rays were divided into two groups according to their root formation. Group A consisted of 18 X-rays with incomplete root formation, except for the palatine root, before treatment and 18 after treatment. Group B consisted of 20 X-rays where root formation was completed before treatment and 20 after treatment. The root lengths were measured with digital caliper and the registered measures were submitted to the error of the method and statistical analysis, Student $t$ test, to verify the differences regarding the root length before and after the treatment with occipital headgear. Results: In group A, there was a significant increase of the root lengths, while in the group B the differences were not significant. Posttreatment Group A showed no significant differences with the mean root lengths of pretreatment group $B$, in other words, teeth with incomplete root formation at onset of orthodontic treatment presented normal root growth during the active treatment. Conclusion: Therefore, it was concluded that high-pull headgear didn't influence negatively in the root formation and it didn't provoke apical resorption of the molars submitted to the action of the appliance, suggesting that occipital headgear doesn't present risks to the root structure and formation when correctly indicated and applied.
\end{abstract}

Keywords: Apical root resorption. Headgear appliance. Dental Class II, division 1 malocclusion.

\section{REFERÊNCIAS}

1. AL-QAWASMI, R. A.; HARTSFIELD JR., J. K.; EVERETT, E. T.; FLURY, L.; LIU, L.; FOROUD, T. M.; MACRI, J. V.; ROBERTS, W. E. Genetic predisposition to external apical root resorption in orthodontic patients: linkage of chromosome-18 marker. J. Dent. Res., Alexandria, v. 82, no. 5, p. 356-360, May 2003.

2. BREZNIAK, N.; WASSERSTEIN, A. Orthodontically induced inflammatory root resorption. Part I: The basic science aspects. Angle Orthod., Appleton, v. 72, no. 2, p. 175-179, Apr. 2002.

3. BREZNIAK, N.; WASSERSTEIN, A. Orthodontically induced inflammatory root resorption. Part II: The clinical aspects. Angle Orthod., Appleton, v. 72, no. 2, p. 180-184. Apr. 2002.

4. BRIN, I. et al. External apical root resorption in Class II malocclusion: a retrospective review of 1-versus 2-phase treatment. Am. J. Orthod. Dentofacial Orthop., St. Louis, v. 124, no. 2, p. 151-156, Aug. 2003.

5. CHEN, W.; CHEN, R. J. Effect of high pull J hook headgear on root resorption during orthodontic treatment. Shanghai Kou Qiang Yi Xue, Shanghai, v. 14, no. 4, p. 345-347, Aug. 2005.

6. GHOLSTON, L. R.; MATTISON, G. D. An endodonticorthodontic technique for esthetic stabilization of externally resorbed teeth. Am. J. Orthod., St. Louis, v. 83, no. 5, p. 435-440, May 1983.
7. HAMILTON, R. S.; GUTMANN, J. L. Endodontic-orthodontic relationships: a review of integrated treatment planning challenges. Int. Endod. J., Oxford, v. 32, no. 5, p. 343-360, Sept. 1999.

8. HARRIS, E. F. Root resorption during orthodontic therapy. Semin. Orthod., Philadelphia, v. 6, no. 3, p. 183-194, Sept. 2000.

9. HENDRIX, I.; CARELS, C.; KUIJPERS-JAGTMAN, A. M.; VAN'T HOF, M. A radiographic study of posterior apical root resorption in orthodontic patients. Am. J. Orthod. Dentofacial Orthop., St. Louis, v. 105, no. 4, p. 345-9, Apr. 1994.

10. HOLLENDER, L.; RONNERMAN, A.; THILANDER, B. Root resorption, marginal bone support and clinical crown length in orthodontically treated patients. Eur. J. Orthod., Oxford, v. 2, no. 4, p. 197-205, 1980.

11. HOUSTON, W. J. The analysis of errors in orthodontic measurements. Am. J. Orthod., St. Louis, v. 83, no. 5, p. 382-390, May 1983.

12. LEVANDER, E.; MALMGREN, O. Evaluation of the risk of root resorption during orthodontic treatment: a study of upper incisors. Eur. J. Orthod., Oxford, v. 10, no. 1, p. 30-38, Feb. 1988. 
13. LINGE, B. O.: LINGE, L. Apical root resorption in upper anterior teeth. Eur. J. Orthod., Oxford, v. 5, no. 3, p. 173-183, Aug. 1983.

14. MALMGREN, O.; GOLDSON, L.; HILL, C.; ORWIN, A.; PETRINI, L.; LUNDBERG, M. Root resorption after orthodontic treatment of traumatized teeth. Am. J. Orthod., St. Louis, v. 82, no. 6, p. 487-491, Dec. 1982.

15. MARTINS, D. R.; CANSANÇÃO, J. M.; SANCHEZ, J. F. Avaliação radiográfica de reabsorção radicular, consecutiva ao tratamento ortodôntico (cinco anos após a remoção do aparelho). Ortodontia, São Paulo, v. 27, n. 3, p. 4-8, set./dez. 1994.

16. MASSLER, M.; MALONE, A. J. Root resorption in human permanent teeth: a roentgenographic study. Am. J. Orthod., St. Louis, v. 40, p. 619-633, 1954.

17. MAVRAGANI, M. VERGARI, A.: SELLISETH, N. J.: BOE, O. E.; WISTH, P. L. A radiographic comparison of apical root resorption after orthodontic treatment with a standard edgewise and a straight-wire edgewise technique. Eur. J. Orthod., Oxford, v. 22, no. 6, p. 665-674, Dec. 2000.

18. MOYERS, R. E. Ortodontia. 4. ed. Rio de Janeiro: Guanabara Koogan, 1991.

19. NGAN, D. C.; KHARBANDA, O. P.; BYLOFF, F. K.; DARENDELILER, M. A. The genetic contribution to orthodontic root resorption: a retrospective twin study. Aust. Orthod. J. Sydney, v. 20, no. 1, p. 1-9, May 2004.

20. NOLLA, C. M. The development of the permanent teeth. J. Dent. Child., Fulton, v. 27, p. 254-266, 1960.

21. ODENRICK, L.; BRATTSTROM, V. Nailbiting: frequency and association with root resorption during orthodontic treatment. Br. J. Orthod., London, v. 12, no. 2, p. 78-81, Apr. 1985.

22. OPACIC-GALIC, V.; ZIVKOVIC, S. Frequency of the external resorptions of tooth roots. Srp. Arh. Celok Lek., Belgrade, v. 132, no. 5-6, p. 152-6, May/June 2004.

23. REITAN, K. Initial tissue behavior during apical root resorption. Angle Orthod., Appleton, v. 44, no. 1, p. 68-82, Jan. 1974.

24. REITAN, K. Some factors determining the evaluation of forces in Orthodontics. Am. J. Orthod., St. Louis, v. 43, no. 1, p. 34-45, Jan. 1957
25. REMINGTON, D : JOONDEPH, D. R.: ARTUN, J: RIEDEL, R A.; CHAPKO, M. K. Long-term evaluation of root resorption occurring during orthodontic treatment. Am. J. Orthod.

Dentofacial Orthop., St. Louis, v. 96, no. 1, p. 43-46, July 1989.

26. ROSENBERG, M. N. An evaluation of the incidence and amount of apical root resorption and dilacerations occurring in orthodontically treated teeth having incompletely formed roots at the beginning of Begg treatment. Am. J. Orthod., St. Louis, v. 61, p. 524-525, 1972.

27. RUDOLPH, C. E. An evaluation of root resorption occurring during orthodontic treatment. J. Dent. Res., Alexandria, v. 19, p. 367-371, 1940.

28. SADOWSKI, P. L. Risk assessment and management during clinical Orthodontics. Semin. Orthod., Philadelphia, v. 8, no. 4, p. 185-260, 2002.

29. SHARPE, W.; REED, B.; SUBTELNY, J. D.; POLSON, A. Orthodontic relapse, apical root resorption, and crestal alveolar bone levels. Am. J. Orthod. Dentofacial Orthop., St. Louis, v. 91, no. 3, p. 252-258, Mar. 1987.

30. SILVA FILHO, O. G. et al. Estimativa da reabsorção radicular em 50 casos ortodônticos bem finalizados. Ortodontia, São Paulo, v. 26, n. 1, p. 24-36, jan./abr. 1993.

31. SMALE, I.; ARTUN, J.; BEHBEHANI, F.; DOPPEL, D.; VAN'T HOF, M.; KUIJPERS-JAGTMAN, A. M. Apical root resorption 6 months after initiation of fixed orthodontic appliance therapy. Am. J. Orthod. Dentofacial Orthop., St. Louis, v. 128, no. 1 p. 57-67, July 2005.

32. SPURRIER, S. W.; HALL, S. H.; JOONDEPH, D. R.; SHAPIRO, P. A.; RIEDEL, R. A. A comparison of apical root resorption during orthodontic treatment in endodontically treated and vital teeth. Am. J. Orthod. Dentofacial Orthop., St. Louis, v. 97, no. 2 , p. 130-134, Feb. 1990

33. VONDERAHE, G. Post-retention status of maxillary incisors with root-end resorption. Angle Orthod., Appleton, v. 43, no. 3, p. 247-255, July 1973.
Endereço para correspondência

Vania C. V. Siqueira

Rua José Corder 87 - Jardim Modelo

CEP: $13.400-010$ - Piracicaba/SP

E-mail: siqueira@fop.unicamp.br 